Eurasian Journal of Physics and Functional Materials

$2021,5(4), 211-217$

\title{
Development of parameters for the production of biomass and biohydrogen from brewer's grain
}

\author{
A. Toleugazykyzy ${ }^{*}, 1$, D. Tlevlessova ${ }^{2}$, \\ A. Samadun ${ }^{2}$, R. Bekbayeva ${ }^{3}$ \\ ${ }^{1}$ S.Seifullin Kazakh agrotechnical university, Nur-Sultan, Kazakhstan \\ ${ }^{2}$ Almaty Technological University, Almaty, Kazakhstan \\ ${ }^{3}$ Shakarim University, Semey, Kazakhstan \\ E-mail: akerke_toleugazykyzy@mail.ru
}

DOI: $10.32523 /$ ejpfm.2021050405

Received: 28.11.2021 - after revision

\begin{abstract}
In this work, suitable pretreatment conditions have been studied to increase hydrogen production by dark fermentation of brewer's grain (BG). All samples with different concentrations of raw materials were tested: treatment with sulfuric acid with a concentration of $1.5 \%$, autoclaving at $121^{\circ} \mathrm{C}$, purification from impurities by filtration, centrifugation and calibration of the $\mathrm{pH}$ of the medium to 7.5 units. The choice of acid hydrolysis is due to the fact that this type of pretreatment is the most suitable for the further commercialization of this technology. Also, pretreatment performs the task of suppressing methanogens and creating conditions for the life of hydrogen-producing bacteria. Experiments were carried out under mesophilic conditions $\left(37^{\circ} \mathrm{C}\right)$ using wild-type and multiple mutant E. coli. The highest sugar yields were obtained at a $4 \%$ concentration of brewer's grains and in the presence of a concentration of $1.5 \%$ sulfuric acid in the original substrate. The results of the experiments showed that brewer's grains are a valuable product as a source of carbon and energy for microorganisms in the production of biohydrogen, as well as for the production of biomass for further production of value-added products.
\end{abstract}

Keywords: lignocellulosic waste, sugars, dark fermentation, biohydrogen, biomass.

\section{Introduction}

The biomass in lignocellulosic waste is used as a source of carbon and energy by various microorganisms under anaerobic conditions, leading to biohydrogen 
forming due to dark fermentation. Several essential metabolites such as acetic acid, lactic acid, ethanol and amino acids are also produced in a process used by methanogenic bacteria that coexist in these habitats. In their natural habitat, producers and consumers of hydrogen exist together; hence, net hydrogen production has not been detected [1-3].

The hydrogen production by dark fermentation is carried out in the acidogenic phase of the anaerobic process. The methanogenic phase is suppressed to prevent hydrogen consumption. In other words, the substrate is converted to organic acids, preferably acetic and butyric acid. Then no further conversion of these organic acids to methane or $\mathrm{CO}_{2}$ is required. The potential for hydrogen formation during dark fermentation is estimated from the yield of formation as $\mathrm{mol} \mathrm{H}_{2} / \mathrm{mol}$ glucose. Theoretically, conversion of 1 mole of glucose gives 12 moles of hydrogen gas $\left(\mathrm{H}_{2}\right)$. According to the stoichiometry of the reaction, the bioconversion of 1 mol of glucose to acetate gives $4 \mathrm{~mol} \mathrm{H}_{2} / \mathrm{mol}$ glucose, but only $2 \mathrm{~mol} \mathrm{H}_{2} / \mathrm{mol}$ glucose is formed when butyrate is the final product [4].

Before dark fermentation, pretreatment and hydrolysis of the biomass is recommended from both a physiological and a technological point of view. The main goal is to provide enzymatic access to fermentable sugars by disrupting lignin and hemicellulose structures or related chemical bonds.

Most studies on chemical pretreatment of biomass in the literature report dilute acid hydrolysis (AH). Among mineral acids and some volatile fatty acids, $\mathrm{H}_{2} \mathrm{SO}_{4}$ is preferred because it is less aggressive and relatively cheaper $[5,6]$. The acid used in hydrolysis can be in liquid or solid form. Sulfuric acid can be diluted at $100-250^{\circ} \mathrm{C}$ in $0.5-30$ minutes with an acid concentration of $0.5-3 \%$. Concentrated acid hydrolysis usually takes place at room temperature with an acid concentration above $30 \%$. The main disadvantages of AG are toxic byproducts (Harmsen et al. 2010) and excess salt formations (Liu et al. 2013). In addition, reactors that are resistant to corrosion, temperature and pressure are required - these are other factors that should be considered for process simplicity (Taherzadeh and Karimi 2007). Toxic compounds such as 5-HMF and furfural are more produced during dilute AG due to dehydration of hexose (C6) and pentose (C5) sugars at high reaction temperatures (Mosier et al. 2005). Dissolution of biomass can be faster in concentrated AG (Jung et al. 2013); however, the need for high doses of alkali for neutralization results in more salt formation than dilute $\mathrm{AH}$.

The substrate plays an important role in $\mathrm{H}_{2}$ yield, $\mathrm{H}_{2}$ productivity and overall process savings. They mainly depend on the content of carbohydrates in the substrate, bioavailability, and the rate of biodegradation (Chong et al., 2009; Guo et al., 2010; Kapdan and Kargi, 2006; Ntaikou et al., 2010; Ren et al., 2011).). DF studies have made extensive use of carbohydrate-rich substrates, in particular mixtures of pure glucose, sucrose, and starch (Wang and Wan, 2009). But the production of renewable biohydrogen requires the substrate or feedstock to come from renewable resources (Hawkes et al., 2007; Ren et al., 2011). Sources of second generation biomass, such as waste biomass, are numerous and thus may support the supply of renewable substrates for DF (Guo et al., 2010; Kapdan and Kargi, 2006; Show et al., 2012). In addition to biohydrogen and volatile fatty 
acids as valuable by-products, DF also offers biological treatment of organic waste [7-8].

In dark fermentation of glucose as a model substrate, $\mathrm{H}_{2}$-producing bacteria initially convert glucose to pyruvate via glycolytic pathways, producing adenosine triphosphate (ATP) from adenosine diphosphate (ADP) and a reduced form of nicotinamide adenine dinucleotide (NADH) (Li) and Fang, 2007 Pyruvate is further oxidized to acetyl coenzyme A (acetyl-CoA), carbon dioxide $\left(\mathrm{CO}_{2}\right)$ and $\mathrm{H}_{2}$ by pyruvate ferredoxine oxidoreductase and hydrogenase. Depending on the type of microorganism and environmental conditions, pyruvate can also be converted to acetyl-CoA and formate, which can be further converted to $\mathrm{H}_{2}$ and $\mathrm{CO}_{2}$. In addition, acetyl-CoA can be converted to acetate, butyrate and ethanol (Li and Fang, 2007a). Dark fermentation of complex carbohydrates with mixed anaerobic microbiota can produce a wide range of intermediates and by-products depending on operating parameters such as substrate type, substrate loading rate, $\mathrm{pH}$, temperature, and other operating and environmental conditions as they also affect structure microbial community in bioreactors [9].

The low yield of biohydrogen $\left(\mathrm{H}_{2}\right)$ and the limited use of by-products from the dark fermentation of waste biomass limit its large-scale application. Previous studies were aimed at studying the effect of culture $\mathrm{pH}$, substrate concentration, substrate pretreatment and adaptation of the inoculum to $\mathrm{H}_{2}$ yield during dark fermentation of organic biomass waste [10-11]. The results showed that the biodegradability of substrates is important for the selection and application of optimal operating parameters to increase $\mathrm{H}_{2}$ production.

Currently, lignocellulose-containing production wastes are attracting more and more attention from the scientific community because they accumulate on a large scale and can be processed into various products with high added value. Such wastes include brewer's grains. The bulk of carbohydrates in brewer's grains are present in the form of polysaccharides, mainly in the form of lignocellulose, consisting of components of cellulose, hemicellulose and lignin. Obtaining a substrate for microbial fermentation is carried out by means of pre-treatment [12-13].

Pretreatment is a crucial step in transforming complex substrates by microorganisms efficiently. Biological pretreatment (e.g. enzymatic hydrolysis) has been classified as environmentally friendly and has the advantages of low energy consumption, mild processing conditions, and does not create inhibitors. However, enzyme pretreatment is not always practical due to the relatively high cost, long pretreatment time and low efficiency. For example, it has been found that the effect of enzymes (including cellulase and laccase) on increasing biogas production from anaerobic digestion of manure wastes is minimal. By comparison, chemical pretreatment is generally more efficient and easier to scale [14-16].

Most studies of chemical pretreatment of biomass in the literature report dilute acid hydrolysis. Among the mineral acids used for hydrolysis, the most preferred is $\mathrm{H}_{2} \mathrm{SO}_{4}$, since it is less aggressive and relatively cheaper, relatively easily removed from the reaction mixture by precipitation in the form of sulfate, and provides the least destruction of monosaccharides.

In this regard, the production of dark enzymatic biohydrogen can be increased 
by choosing a suitable substrate and optimal pretreatment of the substrate. Consequently, research becomes relevant to establish the optimal working conditions for the hydrolysis of raw materials for further dark fermentation.

\section{Materials and methods}

Brewer's grains from the brewing company LLP "Carlsberg Kazakhstan" (Almaty) were used for testing. At the stage of hydrolysis, the following treatments were applied: treatment with sulfuric acid with different concentrations and duration, autoclaving (at a temperature of $121^{\circ} \mathrm{C}$ ), $\mathrm{pH}$ calibration, filtration and centrifugation.

The essence of the experiments is to study the effect of the acid concentration and the duration of hydrolysis on the yield of sugars and acetic acid. In order to study the yield of sugars, the carbohydrate composition of brewer's grains in native form and after hydrolysis was analyzed. For hydrolysis, dilute sulfuric acid with concentrations from 0.75 to $3 \%$ was used. To obtain the substrate, the concentration of brewer's grains was also varied in the range from $2 \%$ to $20 \%$. Preliminary experiments have shown that $4 \%$ concentration is the most suitable among the selected samples. Different processing conditions showed that the yield of oligosaccharides, monosaccharides and inhibitors depends on the concentration of the acid and the duration of hydrolysis. The most optimal results are considered, where the greatest amount of monosaccharides and the least amount (or absence) of inhibitors were found, since in these values the least destruction of monosaccharides occurs.

The total carbohydrate content was determined using an IRF-454 B2M refractometer. Before starting work, the position of the zero-point of the device was checked with distilled water at a temperature of $20^{\circ} \mathrm{C}$. The upper chamber was opened and the surface of the upper and lower prisms was washed with distilled water, and then the prisms were wiped dry with a clean napkin. With a pipette, two or three drops of distilled water were applied to the plane of the measuring prism (lower chamber) and the upper chamber was closed. The light beam of the illuminator was directed into the window of the upper chamber and the handle with the eyepiece was lowered to the lower position until the border of light and shadow appeared in the field of view, and the crosshair of the grid did not coincide with the border of light and shadow; after adjustment, the prism was wiped dry. The sample was mixed and two or three drops were applied with a glass rod onto a clean and dry prism of the lower chamber of the refractometer, the upper chamber was quickly and smoothly closed, and the counting was carried out immediately. Measurements for each sample were carried out three times; the mean value was isolated from these readings, followed by conversion to sucrose.

The mass concentration of organic acids was determined using a capillary electrophoresis system "KAPEL-105M". The previously prepared hydrolyzate of brewer's grain /alcohol stillage/ molasses with a volume of $2.5 \mathrm{ml}$ was placed in a volumetric flask and diluted in 20-fold ratios using distilled water and 
thoroughly mixed. The diluted sample was filtered through a cellulose acetate filter, after which $1 \mathrm{ml}$ of the sample was transferred into an Eppendorf tube, centrifuged for 5 minutes at $5000 \mathrm{rpm}$. The measurement of organic acids was carried out using a cassette with a capillary $d=75 \mu \mathrm{m}$ and a length of $60 \mathrm{~cm}$. At least three electrophoregrams of each aliquot portion of the sample were recorded and the arithmetic mean of the results of three measurements of organic acids was calculated.

The mass concentration of sugars was determined using an Agilent 1200 HPLC equipped with an Aminex HPX-87P column. Samples were taken $1 \mathrm{~cm}^{3}$ into a medical syringe with a capacity of $5 \mathrm{~cm}^{3}$ through a needle, then the needle was replaced with a filter with a pore diameter of $0.45 \mu \mathrm{m}$ and filtered into a container for liquid samples (vials) with a capacity of $2-6 \mathrm{~cm}^{3}$. Then, chromatographic analysis of the prepared samples was carried out; each sample was analyzed three times. The areas of the sugar peaks were recorded and the calculations were performed using the calibration curves.

\section{Research results and discussion}

The optimal conditions and duration of acid hydrolysis were tested not only by the peak values of the yield of monosaccharides, but also by experimental data during the further production of biohydrogen, i.e. study of inhibitors, rate and cumulative yield of hydrogen production.

Pre-treatment of raw materials and preparation of the substrate. A substrate with a $4 \%$ concentration of brewer's grains was selected for testing. Next, the substrate was diluted with distilled water in two and tenfold ratios, which made it possible to obtain samples with $2 \%$ and $0.4 \%$ concentrations of brewer's grains. All samples were tested: treatment with sulfuric acid $\left(\mathrm{H}_{2} \mathrm{SO}_{4}\right)$ with a concentration of $1.5 \%$, autoclaving at $121^{\circ} \mathrm{C}$, purification from impurities by filtration, centrifugation and calibration of the $\mathrm{pH}$ of the medium to 7.5 units with the use of $\mathrm{KOH}$.

Overnight cultures were prepared using wild type bacteria E. coli BW25113 and multiple mutant that were cultured in peptone medium under anaerobic conditions. On the prepared substrates of brewer's grains, night cultures were introduced. Further, the obtained samples were sent to the stage of microbial fermentation, where an anaerobic condition and a constant temperature of the substrate $\left(37^{\circ} \mathrm{C}\right)$ were ensured.

To determine the ORP of the bacterial medium, a pH/ORP Meter HI 2211 and a pH electrode HI 3131 (HANNA lnstruments, Portugal) were used. In samples with a $4 \%$ concentration ( 0 ) of brewer's pellet, at 6 hours of measurements, the readings of the electrodes decreased to values below $-400( \pm 10 \mathrm{mV})$, which confirmed the release of hydrogen molecules. Also, at 24 and 48 hours of measurements, the evolution of hydrogen continued in the specified sample. In two times diluted samples $(2 x)$ of brewer's grains, the evolution of hydrogen molecules was observed in the 3rd hour of measurements and continued up to 48 hours. In tenfold diluted samples (10x) of brewer's grains, no evolution of 
hydrogen molecules was found.

To measure the $\mathrm{pH}$ of the substrate, a $\mathrm{pH}$ meter $\mathrm{H} 13220 \mathrm{pH}$-selective electrode HI 1131 (Hanna lnstruments, Portugal) was used. In all experimental samples, a significant decrease in $\mathrm{pH}$ was observed. In samples with a $4 \%$ concentration of brewer's grains at 96 hours, the $\mathrm{pH}$ dropped to 5.32, and in a two-fold diluted sample to 5.18, and ten times diluted to a sample of 7.01. Measurement of optical density (0D). The formation of bacterial biomass in substrates was studied using a Cary 60 UV-vis spectrophotometer (Agilent Technologies, USA) by measuring the dynamics of changes in optical density at $600 \mathrm{~nm}$. In general, an increase in optical density was observed in all samples during the entire measurement period. In particular, in the experimental sample with a $4 \%$ concentration of brewer's grains, where hydrogen evolution was observed, the optical density increased from 0.0400 to 0.7317 . Also in the experimental sample, diluted two times, where the evolution of hydrogen molecules was also observed, the optical density increased from 0.0613 to 0.8211 .

In samples with a $4 \%$ concentration of brewer's grains, at 6 hours of measurements, the readings of the electrodes decreased to values below $-400( \pm 10 \mathrm{mV})$, which confirmed the release of hydrogen molecules. Also, at 24 and 48 hours of measurements, the evolution of hydrogen continued in the specified sample. In two times diluted samples of brewer's grains at the 6th hour of measurements, the evolution of hydrogen molecules was observed and continued until the 24th hour. In ten times diluted samples of brewer's grains, the evolution of hydrogen molecules was not detected. In all test samples, a significant decrease in $\mathrm{pH}$ was observed. In samples with a $4 \%$ concentration of brewer's grains at 72 hours, the $\mathrm{pH}$ dropped to 5.66, and in a twice diluted sample to 6.44 and ten times diluted to 6.83. Measurement of optical density. In general, an increase in optical density was observed in all samples during the entire measurement period. In particular, in a prototype with a $4 \%$ concentration of brewer's grains, where hydrogen evolution was observed, the optical density increased from 0.0179 to 0.6343 . Also, in a test sample diluted two times, where hydrogen evolution was observed, the optical density increased from 0.0360 to 0.3136 .

\section{Conclusion}

Experimental data show that as a result of hydrolysis and microbial fermentation of substrates with different concentrations of brewer's grains using wild-type bacteria - E. coli BW25113 and the multiple mutant, optimal conditions and parameters of biochemical and microbiological processes for obtaining molecular hydrogen have been established. It should also be noted that this reproduces biomass, which is an environment rich in various organic substances for further secondary and full use in obtaining products with high added value. 


\section{Acknowledgments}

This research has been funded by the Science Committee of the Ministry of Education and Science of the Republic of Kazakhstan (Grant No. AP09562068).

\section{References}

[1] J.F. Soares et al., Renewable and Sustainable Energy Reviews 117 (2020) 109484. [CrossRef]

[2] A. Singh et al., Energies 8 (2015) 13062-13080. [CrossRef]

[3] O. Sarkar, SV. Mohan, Biofuel Res J 3 (2016) 458-4569. [CrossRef]

[4] K.Y. Show et al., Int J Hydrogen Energy 35 (2010) 13350-13355. [CrossRef]

[5] C.C. Chen, C.Y. Lin, Adv Environ Res 7 (2003) 695-699. [CrossRef]

[6] S. Mirzoyan et al., International Journal of Hydrogen Energy 45(35) (2020) 17233-17240. [CrossRef]

[7] M. Galbe, G. Zacchi, Biomass and Bioenergy 46 (2012) 70-78. [CrossRef]

[8] M.L. Zhang et al., Biomass Bioenergy 31 (2007) 250-254. [CrossRef]

[9] F. Hawkes et al., Int J Hydrogen Energy 32 (2007) 172-184. [CrossRef]

[10] R. Lin et al., Bioresour Technol. 196 (2015) 250-255. [CrossRef]

[11] A. Ciranna et al., Int J. Hydrogen Energy 39 (2014) 6391-6401. [CrossRef]

[12] Y.T. Fan et al., Biomass Bioenergy 30 (2006) 493-496. [CrossRef]

[13] L.J. Jonsson et al., Biotechnol. Biofuels 6 (2013). [CrossRef]

[14] G. Kumar et al., Algal Res 11 (2015) 326-333. [CrossRef]

[15] A.C. Chang et al., Int J Hydrogen Energy 36 (2011) 14280-14288. [CrossRef]

[16] G. Cao et al., Int J. Hydrogen Energy 34 (2009) 7182-7188. [CrossRef] 